\title{
Simulating galaxy clusters : the ICM and the galaxy populations
}

\author{
Alessio D. Romeo* \\ Univ. di Catania, Italy and TAC Copenhagen, Denmark \\ E-mail: arodet.astro.it
}

\section{Jesper Sommer-Larsen}

TAC, Nordita and AO, Copenhagen, Denmark

E-mail: jslarsen@tac.dk

\section{Laura Portinari}

TAC Copenhagen, Denmark and Tuorla Obs, Finland

E-mail: lporti@utu.fi

\begin{abstract}
Cosmological LambdaCDM TreeSPH simulations of the formation and evolution of galaxy groups and clusters have been performed. The simulations include: star formation, chemical evolution with non-instantaneous recycling, metal dependent radiative cooling, strong star burst and (optionally) AGN driven galactic super winds, effects of a meta-galactic UV field and thermal conduction. We report results on the temperature and entropy profiles of the ICM, the X-ray luminosity, cold fraction, M/L and IMLR ratios in gas and stars, metal abundances and gradients. Besides, the properties of the galaxy populations in the two richest clusters are discussed: global star formation rates of the cluster galaxies, the total K-band luminosity, the galaxy luminosity functions at $\mathrm{z}=0$ and their redshift evolution, the colour-magnitude relation ("red sequence") as resulting from metallicity effects, and the role of the IMF in reproducing colours and abundances of the stellar populations. Finally the contribute (20-40\%) to cluster light from the intra-cluster stars and the $\mathrm{cD}$ galaxy has been investigated: surface brightness profiles, mean colours and chemical abundances, kinematics (velocity dispersion and distributions) of the IC stellar populations.
\end{abstract}

Baryons in Dark Matter Halos

5-9 October 2004

Novigrad, Croatia

${ }^{*}$ Speaker. 


\section{Introduction}

Non-gravitational heating and radiative cooling have been extensively recoursed to in the literature in order to complete the basic gravity-only driven scenario describing the thermal behaviour of the ICM, which is unable to reproduce the scaling relations observed between $L_{X}, T$ and $S$.

So far few hydrodynamical simulations have been performed which were able to pursue at the same time and in a self-consistent way the target of realistically modelling the ICM dynamics by including both non-gravitational heating/cooling (complete with feedback/star formation) and metal enrichment directly springing from chemically following up the stellar feedback itself (e.g. Tornatore et al. 2004). Moreover, only recently simulations have reached a level of sophistication adequate to even trace star formation and related effects in individual galaxies, coupled with the chemical enrichment of the ICM by galactic winds.

In this series of papers, we present an analysis of the properties of the galaxy population of clusters as predicted directly from cosmological simulations that follow detailed baryonic physics, gas dynamics and galaxy formation and evolution. The resolution for such $\mathrm{N}-$ body + hydrodynamical simulations cannot reach the extent of resolving galaxies at the faint end of the luminosity function $\left(M_{B} \gtrsim-16\right)$. On the other hand, our simulations aim at describing in a self-consistent way the hydrodynamical response of the ICM to star formation, stellar feedback and chemical enrichment.

Our cosmological N-body simulation has been performed using the FLY code (Antonuccio et al. , 2003) on a standard $\Lambda \mathrm{CDM}$ model The selected clusters and groups isolated from the cosmological simulation, as identifi ed at $z=0$, have been resampled and resimulated (adding the baryon particles according to a $f_{b}=0.12$ ) by means of an improved version of the Tree-SPH code described in Sommer-Larsen, Götz \& Portinari (2003; SLGP). The mass resolution reached inside the resampled lagrangian subvolumes from the cosmological set was such that $m_{D M}=1.8$. $10^{9} h^{-1} M_{\odot}$ and $m_{g}=m_{*}=2.5 \cdot 10^{8} h^{-1} M_{\odot}$.

Most of the results hereby presented refer to the two larger systems: "sub-Coma" $\left(M_{v i r}=\right.$ $\left.12.4 \cdot 10^{14} M_{\odot},<k T_{e w}>\simeq 6 \mathrm{keV}\right)$ and "Virgo" $\left(2.8 \cdot 10^{14} M_{\odot}, 3 \mathrm{keV}\right)$, though four more smaller clusters and groups have been simulated which span the range $1-2 \mathrm{keV}$.

The adopted TreeSPH code schematically includes the following features: conservative solution of entropy equation; metal-depending atomic radiative cooling; star formation, according to either an Arimoto-Yoshii (AY) or a Salpeter IMF; feedback as starburst (SN II) driven galactic winds; chemical evolution with non-instantaneous recycling of gas and heavy elements $(\mathrm{H}, \mathrm{He}, \mathrm{C}$, $\mathrm{N}, \mathrm{O}, \mathrm{Mg}, \mathrm{Si}, \mathrm{S}, \mathrm{Ca}, \mathrm{Fe}$ ); thermal conduction; meta-galactic, redshift-dependent UV fi eld. All the runs implement the "Super-Wind" (SW) scheme, characterized by high effi ciency parameters $\varepsilon_{S F}$ and $f_{\text {wind }}$ : the latter is amplifi ed by a factor 2 or 4 in those runs simulating the effect of stronger non-stellar feedback (AGN). On the top of this model, it has been added either thermal conduction (at $\frac{1}{3}$ Spitzer value (COND runs), or pre-heating in form of $0.75 / 1.50 / 50 \mathrm{~cm}^{2} \mathrm{keV}$ injected per particle at $z=0$ (PH runs). Finally, we ran as a test the WFB simulation, which adopts a normal feedback scheme with only early winds at low values of $f_{\text {wind }}$ (as described in SLGP), and the ADIABATIC run without cooling nor star formation at all. 

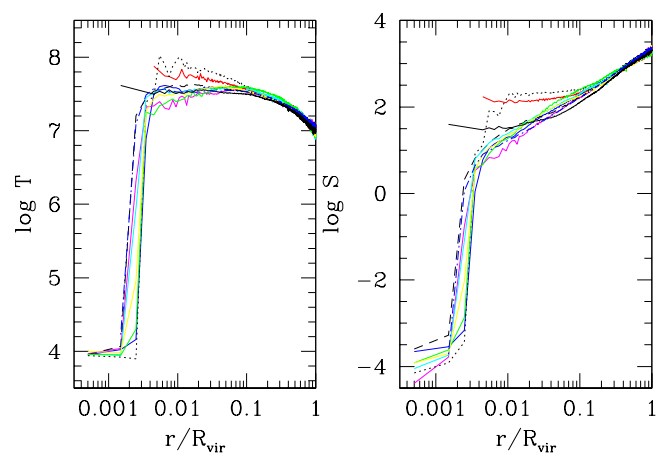

Figure 1: Temperature (left) and Entropy (right) profiles of "Virgo", all models: same colours as in Fig. 3.

\section{Results on the ICM}

\subsection{Thermal properties}

Observations of X-ray surface brightness profi les in poor clusters and groups of galaxies had hinted at the existence of isentropic cores or "entropy floor" at around $100 \mathrm{keV} \cdot \mathrm{cm}^{2}$ (e.g. Ponman et al. , 2003), associated with a reduced gas central density. Systems at all scales, and in particular low-mass ones, show evidence for excess entropy compared to that expected from pure gravitational heating by compression. In Fig. 1 the inner pattern of temperature and entropy for particles in "Virgo" cluster is shown: most models exhibit central entropy values of $\sim 10-20 \mathrm{keV} \cdot \mathrm{cm}^{2}$, which may get agreed with inferred values in cold-core clusters; instead a plateau has formed only in two distinct cases, namely the WFB metal-independent scheme with Salpeter IMF and the 4-times SW scheme with AY IMF. The former has less effi cient cooling and therefore lets more high-entropy gas settle down in a core. The SWx4 model ends up with forming a floor around $100 \mathrm{keV} \cdot \mathrm{cm}^{2}$ at $z=0$.

In Fig. 2 the relation $L_{X}-T$ at $z \simeq 0$ is shown, The observed (open) points exhibit the characteristic double-slope behaviour; as expected, the adiabatic runs lie above this line. Overall, the predicted luminosities are higher than the observed one; both an enhanced feedback mechanism and a top-lighter IMF, however, can indipendently concur in lowering the X-ray emission from the ICM: the latter effect seems to be dominant, since the runs with Salpeter IMF and Weak Feedback, which result in a low level of metal enrichment as well, are those placed lowest. All in all, when strengthening the feedback up to 4 times and using a top-heavy IMF, a satisfactory normalization seems to be reached. One could conclude then that previous simulations successful at reproducing both the L-T relation and the surface brightness profi les and the rather high entropy floor as well, might have underestimated the contribution of metals to radiative cooling by adopting simple primordial cooling functions.

Baryonic mass of clusters is largely dominated by the hot ICM mass, yet it is the (stellar) mass 


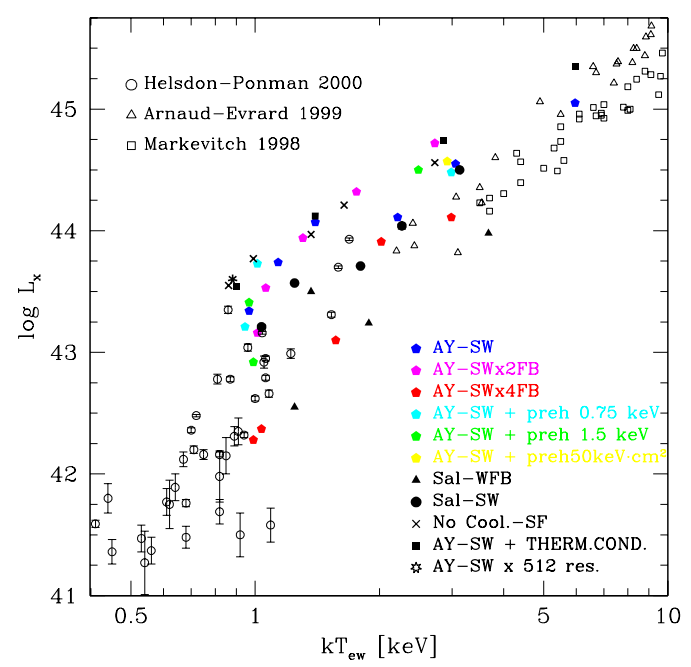

Figure 2: X-ray luminosity - emission weighted temperature scaling relation from the inner $1 \mathrm{Mpc}$.

in galaxies playing the major role to the purpose of chemical enrichment of ICM itself. A measure of galaxy formation effi ciency is the "cold fraction", defi ned as the fraction of baryons in the form of cold gas and stars. It plays a major role in assessing the mechanisms responsible for the "excess entropy": the larger the cold fraction (hence star formation), the more low entropy gas is removed from the core; with a small cold fraction, instead, strong (pre)heating or feedback are needed to enhance the entropy level. The radial profi les of the cold fraction are shown in Fig. 3 for our two largest simulated clusters: the AY-SW simulations (including the conduction and preheating cases) are those that best reproduce the overall level, or equivalently the $\frac{M_{I C M}}{L}$ ratio, within the virial radius. The Salpeter runs instead, especially the weak feedback case, result in too large $f_{c}$, while the enhanced feedback cases predict signifi cantly too low values. It is a remarkable result that our simulations with SN feedback (with a top-heavy IMF, but without invoking additional sources of energy input via enhanced feedback) fairly succeed in reproducing the low fraction of baryons in the cold phase. Indeed it is a common feature of all hydrodynamical simulations with cooling and star formation ending up with a too large fraction of gas to get converted into stars (30 up to 55\%, against observed 10-20\%). When including also non-gravitational (pre)-heating, star formation gets attenuated by preventing gas from cooling at high redshift, hence causing a decrease in the cold fraction.

\subsection{Metal enrichment}

The presence of heavy elements in the hot intra-cluster gas calls for the processing of a signifi cant fraction of the ICM inside galaxies. In our simulations, the super-winds connected to the SNII feedback prescription lead to the enrichment of the ICM by dispersal of the chemical elements produced by the star particles. Fig. 4 shows the simulated iron abundance profi les, compared to data for cool core and non-cold core clusters. Our simulated clusters are best compared to cold-core clusters. Evidently, the simulated gradients are steeper than the observed ones. This seems to be a general problem in simulations, probably related to the fact that star formation is very concen- 


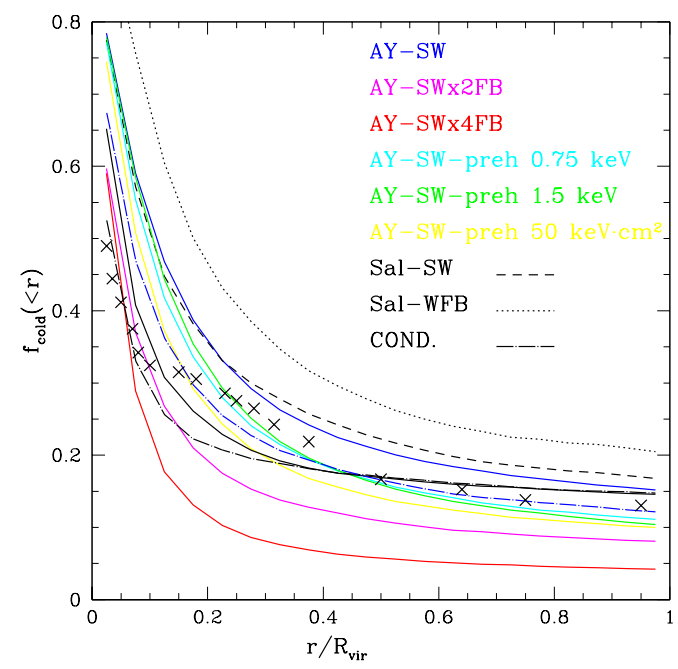

Figure 3: Cold gas plus stellar radial profiles of the two most massive clusters ("Coma": dot-dashed line). Crosses: observed cold fraction profiles, derived from the $\frac{M_{*}}{M_{I C M}}$ data of Roussel et al. (2000).

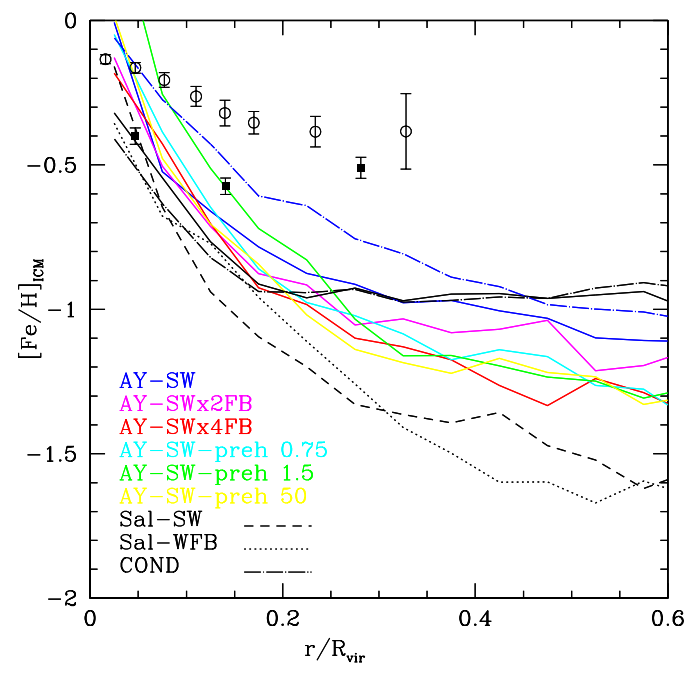

Figure 4: Iron gradients in the ICM of the two most massive simulated clusters. Data from De Grandi et al (2004) refer to cold-core (open circles) and non cold-core (filled squares) clusters.

trated toward the inner region of the $\mathrm{cD}$ galaxy. The ICM is also enriched by $\alpha$-elements, mainly produced by SNII and specifi cally traced by silicon abundances: We found a trend of supersolar $[\mathrm{Si} / \mathrm{Fe}]$ increasing with radius in all our simulations.

Moreover, it has also been found that the overall iron abundance in the ICM of simulated clusters is unvaried from $z=0$ to $z=1$, in very good agreement with observations. Since the bulk of the stars in the cluster are formed at $z \geq 2$, at $z=2$ we predict much lower iron metallicities, but such redshift range is not probed by observations yet. As expected, $[\mathrm{Si} / \mathrm{Fe}]$ decreases in time due to the delayed iron contribution of SNIa with respect to the bulk of the silicon production (from SNII). 

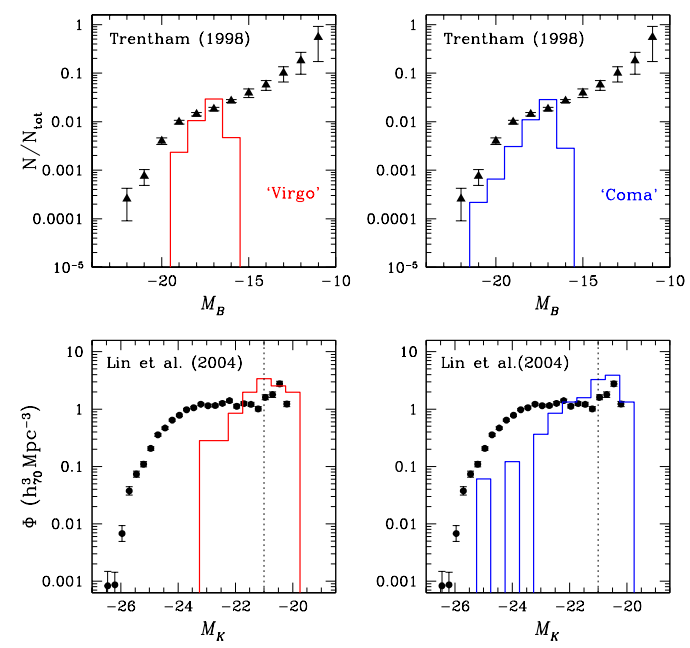

Figure 5: Top panels: B-band luminosity functions at $z=0$ for the standard (AY-SW) "Virgo" (left) and "Coma" (right) simulations, compared with the observed composite LF of Trentham 1998, triangles. Bottom panels: K-band LFs for the same clusters, compared to the observed average LF by Lin et al. (2004); the dotted line marks the limit $M_{K} \leq-21$ where the observational estimate is considered reliable.

\section{Results on cluster galaxies}

\subsection{Luminosity Functions}

Each star particle represents a Single Stellar Population (SSP), with individual stellar masses distributed according to a particular IMF. We keep record of the age and the metallicity of each of these SSPs and hence the global luminosities and colours of the member galaxies are computed. We identify for the standard (SW-AY) runs 42 galaxies in "Virgo" and 212 galaxies in "Coma".

In Fig. 5 we compare the B-band and K-band luminosity function (LF) of our simulated cluster galaxies, exluding the $\mathrm{cD}$, to two observational composite LFs. The number of resolved individual galaxies quickly drops for objects fainter than $M_{B} \sim-17$, both in "Virgo" and in "Coma", due to the resolution limits. Although we are missing the dwarf galaxies that largely dominate in number, we are able to describe the bulk of the stellar mass and of the luminosity in clusters, which is dominated by galaxies around $L_{*}$ In the signifi cative magnitude range, the shape of the predicted and observed LF is directly comparable. Yet, the simulated LF is steeper than the observed one, namely we underestimate the relative number of bright galaxies, at least within the SW model here shown. We have found that increasing the feedback strength results in decreasing the masses of all galaxies, and hence, in particular, the number of bright galaxies. Alongside this, the LF in the Salpeter cases is broader in luminosity, due the lower stellar feedback with respect to the AY IMF, allowing a larger accumulation of stellar mass in galaxies.

In Fig. 6 we show the evolution of the B and K band LFs of our "Virgo" and "Coma" clusters. The $z=1 \mathrm{LF}$ is shifted to brighter magnitudes with respect to the distribution at $z=0$. Both passive luminosity evolution and dynamical mass evolution are seen to play a role. In particular, since the bulk of the stars in our galaxies are formed at $z \gtrsim 2$ (see Fig. 7, bottom panel), luminosity dimming is an important effect. 

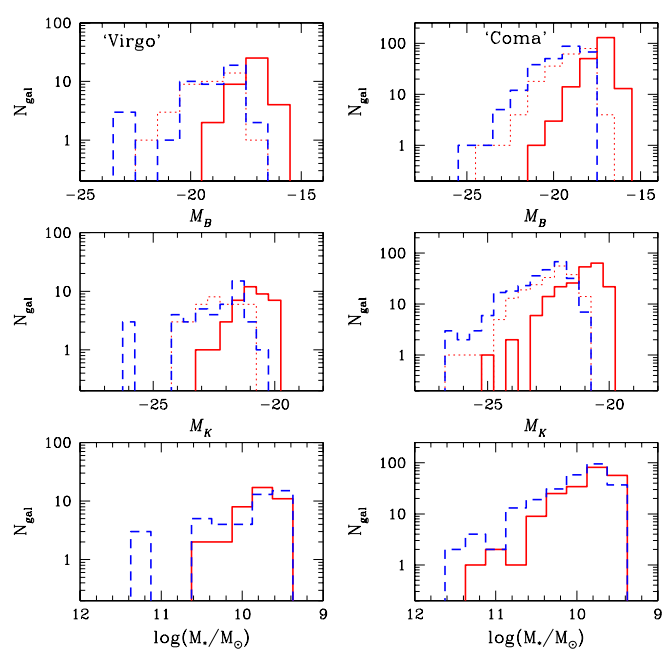

Figure 6: Top panels: Luminosity functions in absolute number of galaxies per $B$-band magnitude bin for the standard (AY-SW) "Virgo" and "Coma" simulations, at $z=0$ (solid lines) and $z=1$ (dashed lines); the dotted lines represents the expected $z=1 \mathrm{LF}$ if pure passive evolution of the stellar populations is applied to the $z=0$ LF. Middle panels: Same, for the K-band luminosity functions. Bottom panels: Mass function of cluster galaxies at $z=0$ and $z=1$.

The LF inferred at $z=1$ as expected from pure passive evolution of the $z=0 \mathrm{LF}$ (dotted line in Fig. 6) matches very closely the actual LF in the $z=1$ frame. This indicates that luminosity dimming of the stellar populations drives most of the LF evolution and the fading to fainter magnitudes from $z=1$ to $z=0$ for the simulated galaxy population. In the brightest luminosity bins of our LF, some additional, dynamical effect seems to be required. To assess mass evolution effects, we plot in the lower panels of Fig. 6 the mass function of cluster galaxies at $z=1$ and at $z=0$, which appear indeed very similar. "Over-merging" onto the central $\mathrm{cD}$ in cosmological simulations can be responsible for depleting the number of galaxies at the bright end of the LF (Springel et al. 2001). Yet the results of a test simulation at 8 times higher mass and two times better force resolution we performed do allow us to disregard this interpretation.

\subsection{The Red Sequence}

The light and stellar mass in clusters of galaxies is dominated by bright, massive ellipticals, which are known to form a tight colour-magnitude relation, or Red Sequence (Gladders et al. 1998). In Fig. 7ab, we compare the colour-magnitude relation for our "Coma" cluster galaxies, excluding the cD, to two observed Red Sequences of Coma. There is overall agreement, although our Red Sequence is not as extended as the observed one, and it also displays a larger scatter. The colourmagnitude relation is classically interpreted as a mass-metallicity relation. The bulk of the stars in the simulated galaxies are formed at $z \gtrsim 2$, hence our galaxies are essentially coeval (Fig. 7d) and their colour-magnitude relation is mostly a metallicity effect, as it can be seen from combining these results with the metallicity-luminosity relation displayed in Fig. 7c.

Moreover, the simulated Red Sequence appears to be a robust prediction of our simulations, quite unaffected by the adopted physical prescriptions other than the chosen IMF. 


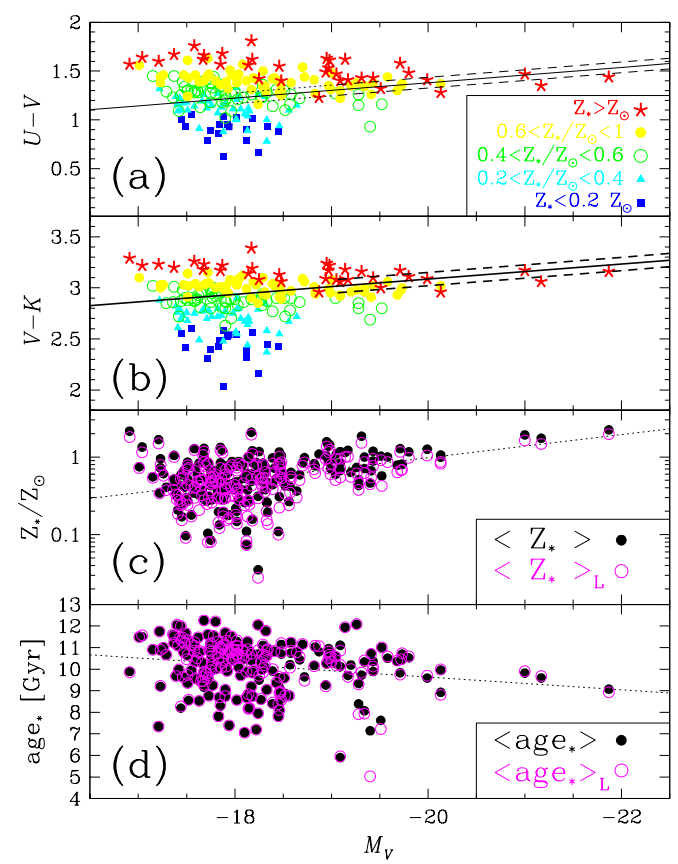

Figure 7: (a-b) Colour-magnitude relation at $z=0$ for galaxies in the simulated "Coma" cluster, compared to the observed relation with its scatter (solid and dashed lines, from Bower et al. 1992; dotted lines, from Terlevich et al. 2001). The extension of the dashed/dotted lines indicates the magnitude range actually probed observationally, while the solid line is an extrapolation of their fit to lower magnitudes. (c) Metallicityluminosity relation for the "Coma" galaxies (full symbols for mass-averaged stellar metallicity, open symbols for luminosity-weighted metallicity); the dotted line is a linear fit. (d): Age-luminosity relation for the "Coma" galaxies (mass-average and luminosity-weighted stellar ages); the dotted line is a linear fit.

\section{References}

[1] Antonuccio-Delogu V., Becciani U., Ferro D., 2003, Comput. Phys. Commun. 155, 159

[2] De Grandi S., Ettori S., Longhetti M., Molendi S., 2004, A\&A 419, 7

[3] Gladders M.D., Lopez-Cruz O., Yee H.K.C., Kodama T., 1998, ApJ 501, 571

[4] Lin Y., Mohr J., Stanford S.A., 2003, ApJ 591, 749

[5] Ponman T.J., Sanderson A.J.R., Finoguenov A., 2003, MNRAS 343, 331

[6] Portinari L., Moretti A., Chiosi C., Sommer-Larsen J., 2004, ApJ 604, 579

[7] Romeo A.D., Portinari L., Sommer-Larsen J., 2004, MNRAS submitted, astro-ph/0404445 (Paper II)

[8] Roussel H., Sadat R., Blanchard A., 2000, A\&A361, 429

[9] Sommer-Larsen J., Götz M., Portinari L., 2003, ApJ 596, 47 (SLGP)

[10] Sommer-Larsen J., Romeo A.D., Portinari L., 2004, MNRAS in press, astro-ph/0403282 (Paper III)

[11] Tornatore L., Borgani S., Matteucci F., Recchi S., Tozzi P., 2004, MNRAS 349, L19

[12] Trentham N., 1998, MNRAS 295, 360 